\title{
Number Crunching the Beautiful Game
}

\author{
A stochastic model that analyzes the movements of a handful of players \\ correctly reproduces the statistics of nearly 2000 real-life soccer games.
}

\author{
By Marric Stephens
}

$\square$ ata-analysis techniques have made professional basketball and baseball much more measurement driven. The approach has yet to make headway in the game of soccer, however, possibly because, in soccer, a team's performance depends on player behaviors that are difficult to quantify. Andrés Chacoma, at the Enrique Gaviola Institute of Physics, Argentina, and colleagues hope to change that with their new model, which applies number-crunching methods to the "beautiful game" [1]. Eventually, Chacoma says, soccer coaches might use such models to design and analyze training sessions.

Chacoma and colleagues used a newly released dataset comprising millions of in-game events from the 2017-2018 season of five European soccer leagues. Focusing on switches of ball possession between teams, the researchers found that tackles and passes dominate the action, and that most periods of possession involve just two or three players. Thus, the researchers created a model in which two players attacking the

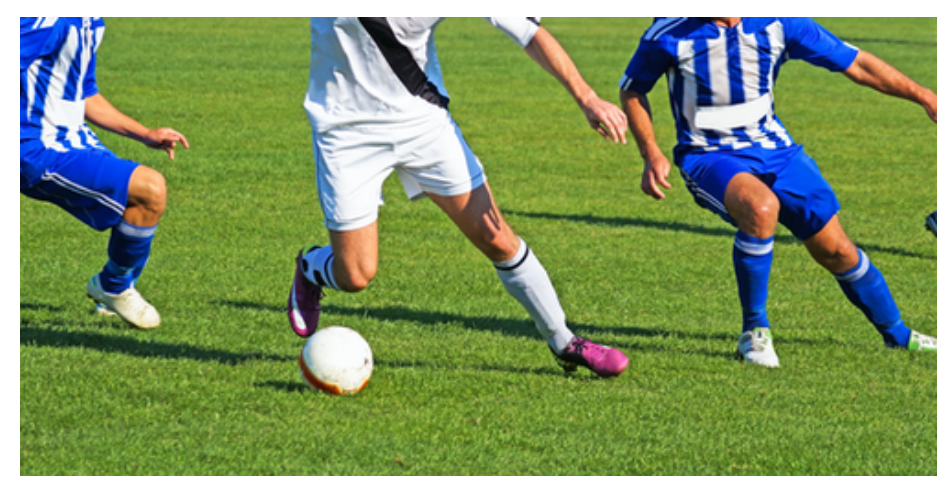

Credit: majorosl66/stock.adobe.com goal pass the ball back and forth while a defender tries to take possession.

The group found that the model produced possession periods whose statistics-period length, pass distance, and the number of passes performed-had distributions very similar to those in the real dataset. They also found that the dynamics underlying this similarity were captured by an even simpler description of the game. This simpler model represents the defender as a random walker in $1 \mathrm{D}$, and the defender's pursuit of the ball as a directional bias applied to each of the defender's step. The possession interval ends when the defender reaches an absorbing barrier (the ball) at one end of the line. The researchers are considering extending their model to include additional players and are also adapting it to include a preferential direction on the player's movements.

Marric Stephens is a Corresponding Editor for Physics based in Bristol, UK.

\section{REFERENCES}

1. A. Chacoma et al., "Modeling ball possession dynamics in the game of football," Phys. Rev. E 102, 042120 (2020). 